\title{
Nanodiscs for structural and functional studies of membrane proteins
}

\author{
Ilia G Denisov ${ }^{1}$ \& Stephen G Sligar ${ }^{1,2}$
}

\begin{abstract}
Membrane proteins have long presented a challenge to biochemical and functional studies. In the absence of a bilayer environment, individual proteins and critical macromolecular complexes may be insoluble and may display altered or absent activities. Nanodisc technology provides important advantages for the isolation, purification, structural resolution and functional characterization of membrane proteins. In addition, the ability to precisely control the nanodisc composition provides a nanoscale membrane surface for investigating molecular recognition events.
\end{abstract}

Membrane proteins (MPs) are central to life processes. They are the key conduit for communication between cells, conduct the vital transformations that produce energy, provide channels for the transport of molecules between the inside and outside of cells and intracellular compartments and are the workhorses for a plethora of enzymecatalyzed metabolic transformations. Because of their role in the regulation of vital cellular functions, MPs are the target of the majority of currently marketed therapeutics. Despite these crucial roles, MPs have been notoriously difficult to work with because they often display altered or loss of activity and function outside of a phospholipid environment. Understanding the molecular mechanisms of MP action is impossible without detailed knowledge of their structure and how they interact with other proteins, nucleic acids and lipids. Thus, the structural biology of MPs occupies a central place in current biophysics, biochemistry and cell biology investigations.

Ideally, an MP structural study should elucidate not only the conformations and protein-protein interactions in supramolecular complexes but also their topology in the lipid bilayer and the details of the protein-lipid interface, which often are highly specific with respect to lipid composition. For many systems and projects, a planar bilayer model system of $\sim 10 \mathrm{~nm}$ in diameter would be ideal, providing space for one or more MPs and allowing access to both sides for the assay of signaling events. By providing such a native membrane environment, nanodiscs have proven to be an invaluable tool for revealing the structure and function of isolated MPs as well as their complexes with other proteins and lipids.

${ }^{1}$ Department of Biochemistry, University of Illinois at Urbana-Champaign, Urbana, Illinois, USA. ${ }^{2}$ Department of Chemistry, University of Illinois at Urbana-Champaign, Urbana, Illinois, USA. Correspondence should be addressed to S.G.S. (s-sligar@illinois.edu).

Received 3 December 2015; accepted 24 February 2016; published online 7 June 2016; doi:10.1038/nsmb.3195
Nanodiscs are discoidal lipid bilayers of 8-16 $\mathrm{nm}$ in diameter, which are stabilized and rendered soluble in aqueous solutions by two encircling amphipathic helical protein belts, termed membrane scaffold proteins ${ }^{1,2}$. The size of nanodiscs is determined by the length of the membrane scaffold protein and the stoichiometry of the lipids used in the self-assembly process. The resultant discoidal bilayers can be made homogeneous and monodisperse and can be obtained with high yield ${ }^{2,3}$.

A large body of published work has used nanodiscs as a vehicle to incorporate recalcitrant MP targets into the bilayer to preserve MP structure and activity. MPs of many types and topologies have successfully been self-assembled into nanodiscs, by starting from a detergent-solubilized mixture including all components: target, lipid mixture of choice and membrane scaffold proteins (Fig. 1). The molar stoichiometric ratio of lipid to scaffold protein is important for optimized yield ${ }^{2,3}$, whereas the size and ratio of scaffold protein to MP may be selected to favor incorporation of predominantly monomeric (in which there is a large excess of scaffold protein and lipid) or oligomeric target into nanodiscs ${ }^{3,4}$. This simple approach provides control over the composition and homogeneity of the resultant assembly and can be optimized to maximize the yield of MP incorporation. If the MP under investigation is unstable to purification in detergent, or a library of all MPs in a specific tissue is desired, incorporation into nanodiscs can be achieved through direct solubilization of cell membranes by using detergents ${ }^{5}$ or membrane-active polymers ${ }^{6,7}$ (Fig. 1, route 2 ). This pathway allows the purification step to be bypassed and accelerates the entire process of nanodisc self-assembly, which is sometimes critically important for preservation of the native and functional form of the target MP (illustrated in Fig. 1). For successful incorporation of purified MPs (Fig. 1, route 1), the most important factor is efficient solubilization and prevention of aggregation of the target protein; these conditions can be realized by using excess lipid and scaffold protein while maintaining the correct overall stoichiometry ${ }^{3}$. Parameters that control the branch point that leads to successful incorporation of a target MP include the choice of detergent, the speed of detergent removal and the identities of the lipid and target ${ }^{3}$.

The number of laboratories using nanodiscs is growing rapidly, as evidenced by the published literature. Nanodiscs are robust and can be frozen or lyophilized with or without an incorporated $\mathrm{MP}^{8}$, and they allow for precise control over the lipid composition. Scaffold proteins are available with a wide range of specific tags for isolation, in vivo targeting, imaging and reversible or irreversible surface immobilization. Nanodiscs are increasingly being used as 'cassettes' that allow an MP to be assayed without denaturation through a variety of analytical 
Figure 1 Assembling MPs into nanodiscs. The standard method for self-assembling an MP into a nanodisc is shown in route 1 (left): after detergent solubilization and purification, the target MP (green) is mixed with the membrane scaffold protein (MSP, blue) and lipids at the correct stoichiometry, followed by detergent removal though incubation with hydrophobic beads. Often, however, the MP is not stable in detergent for the extended times needed for purification. Alternatively (route 2 , right), the starting membrane or tissue can be directly solubilized with excess lipid and scaffold protein and rapid detergent removal, resulting in placement of the target MP (green), together with other MPs (gray) in the tissue, into the nanodisc. Subsequent purification, often with an affinity tag, is performed, and the target is stabilized in the nanodisc environment. This latter route can also be used to generate a soluble MP library that faithfully represents the MPs in the starting tissue.

methods, including surface plasmon resonance ${ }^{9-11}$, electrochemistry and optical waveguides. A partial compendium of nanodisc uses in the study of MP is presented in Table 1, with a focus on structural investigation. Successful approaches include X-ray crystallography, EM, various spectroscopic methods, single-molecule studies, smallangle X-ray scattering (SAXS) and small-angle neutron scattering (SANS). The processes illustrated often take advantage of the ability of nanodiscs to provide a homogeneous sample with a desired target oligomerization state or supramolecular complex topology. Many studies have utilized nanodiscs to control lipid, fatty acid or cholesterol composition; to investigate protein-membrane interaction specificity; or to achieve surface immobilization for sensing and detection, through the use of affinity tags on nanodiscs.

Nanodiscs offer substantial advantages over more classical approaches. For example, MP solubilization in proteoliposomes often results in turbid and viscous samples, which may be especially troublesome in cell-free expression systems ${ }^{12}$ and in many biophysical methods. Nanodisc preparations remain fluid and intact, even at the high concentrations needed for NMR experiments ${ }^{13-18}$. Nanodiscs do not suffer from the well-documented perturbation of MP native structure under non-native conditions, i.e., when present

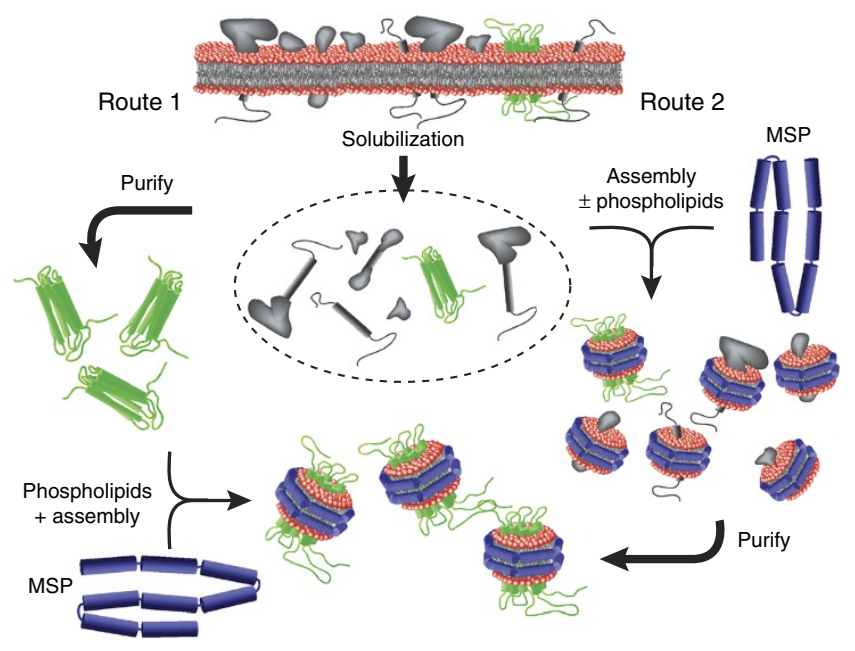

in detergent micelles ${ }^{19}$, and they offer the advantage of maintaining higher structural stability and much longer lifetime than do bicelles. The high kinetic stability of nanodiscs assembled on transmembrane fragments of large MPs and their complexes has proven very useful in the prevention of aggregation during sample preparation for EM studies ${ }^{20,21}$ (Fig. 2). Recently, various amphipathic polymers, for example, SMALPs ${ }^{6}$ or amphipols ${ }^{7}$, have been successful in solubilizing MPs from tissue and forming soluble particles on a nanoscale. Although these polymers are useful for MP extraction, in general, they do not provide the same control over size and composition as do nanodiscs.

\section{Structural biology with nanodiscs}

Several recent studies have provided examples of the expanding use of nanodisc technology in structural biology. Nanodiscs are often used for the isolation and purification of MPs for structural studies, including $\mathrm{X}$-ray crystallography 22,23 and $\mathrm{EM}^{20,21}$. It is often challenging to collect

Table 1 Methods and tools used with MPs incorporated into nanodiscs

\begin{tabular}{|c|c|c|c|}
\hline Features and applications & Experimental methods & Membrane proteins & References \\
\hline \multirow[t]{6}{*}{ Solubilization and stabilization } & X-ray crystallography & GPCR & 23 \\
\hline & NMR & Various MPs & $8,14-18$ \\
\hline & Resonance Raman & Cytochrome P450 & 56 \\
\hline & Optical absorption & Cytochrome P450 and rhodopsins & $53-57$ \\
\hline & EPR & Transporters and rhodopsin & 36,37 \\
\hline & Cell-free expression & Various & 12,42 \\
\hline $\begin{array}{l}\text { Surface immobilization using affinity tags } \\
\text { on scaffold protein or lipids }\end{array}$ & $\begin{array}{l}\text { Single-molecule studies, SPR, LSPR and } \\
\text { sensing and detection }\end{array}$ & Cytochrome P450, GPCR and Factor VII & $9-11,47-50$ \\
\hline Isolation of monomers, oligomers and & Structural and functional studies & Receptors and rhodopsins & $4,53-55,59,60$ \\
\hline functional complexes of multiple proteins & & Cytochrome P450 with CPR and ATPase & $57,58,61,67$ \\
\hline \multirow[t]{2}{*}{ Structurally homogeneous preparations } & SAXS and SANS & Cytochrome $\mathrm{P} 450$ and reductase & $4,42,43,45$ \\
\hline & EM & Receptors and channels & $20,21,26-31$ \\
\hline Fractionation by size, charge or density & $\begin{array}{l}\text { Chromatography, electrophoresis and } \\
\text { ultracentrifugation }\end{array}$ & Various & $3,4,15,59,60$ \\
\hline Protein-membrane binding & SPR & K-Ras protein, TF-VIIa and PgP transporter & $9-11$ \\
\hline \multirow[t]{3}{*}{ Membrane effects on protein properties } & Redox potential & Cytochrome $\mathrm{P} 450$ reductase & 39 \\
\hline & Kinetics & Factor VIII & 29 \\
\hline & & Respiratory complex & 40 \\
\hline
\end{tabular}

SPR, surface plasmon resonance; LSPR, localized SPR; CPR, NADPH-cytochrome P450 reductase. 
Figure 2 Examples of MP structure in nanodiscs. (a) The structure of the ryanodine receptor in a nanodisc, resolved to $\sim 6.1 \AA$ through cryo-EM. The nanodisc is shown as a light-gray envelope with

24 transmembrane helices forming a square structure similar to that of the incorporated voltage-gated sodium channel $\mathrm{Na}_{\mathrm{v}}$ (reproduced with permission from ref. 21, Nature Publishing Group). (b) The structure of KRas4b bound to the surface of a nanodisc, as determined by NMR spectroscopy (PDB 2MSC) ${ }^{38}$.

enough material for structure determination if an MP is unstable in detergent or lipids are absent. Self-assembly of an MP target into nanodiscs, in which the MP is stable and remains in its native conformation, offers a way to accumulate and store material ${ }^{23}$. Clearly, any type of compositional diversity leads to additional complications in structural studies, often prohibiting successful analysis at high resolution. Nanodiscs with incorporated MPs can be generated with controlled composition and a very high degree of size homogeneity ${ }^{2-4}$. Nevertheless, a complete X-ray structure of an MP in a nanodisc bilayer has yet to be reported. A potential challenge is the translational and azimuthal mobility of the incorporated target, which may lead to difficulties in crystallization. Such motion could be potentially be reduced by association with a bifunctional antibody that recognizes both the target and membrane scaffold proteins ${ }^{24}$ or by reducing the size of the nanodisc by shortening the scaffold-protein belt and using less phospholipid. For an MP with large globular domains on either side of the bilayer, crystal contacts might potentially be made between the target MP, thus spectacularly rendering the nanodisc blurred but revealing the structure of the MP in a bilayer.

Electron microscopy. Recent advances in EM have brought nearatomic resolution to the structural biology tool kit. Because of their high homogeneity, nanodiscs are being widely used in EM studies of isolated MPs and functional MP complexes ${ }^{20,25-28}$ as well as MP binding on the surface of the bilayer ${ }^{29,30}$. Three-dimensional reconstructions, based on combined analysis of multiple $2 \mathrm{D}$ images, have been reported $21,27,31$. The incorporation of an MP or membrane domain(s) into nanodiscs prevents aggregation of the MP and significantly improves the sample quality ${ }^{20}$. Atomic-level detail is rapidly becoming a reality, as evidenced by the recent structure of the ryanodine receptor in nanodiscs ${ }^{21}$ (at a resolution of $6.1 \AA$ ), in which the positions of individual transmembrane helices are resolved, and the 3.8-A-resolution structure of an $\mathrm{Mg}^{2+}$ channel by Subramaniam and colleagues ${ }^{31}$.

NMR and EPR methods. One of the most useful advantages of nanodiscs for structural studies of MPs is that they enable work at high concentrations without aggregation or loss of integrity, which are often problematic in using the powerful tools of NMR for structural determination. The first NMR spectra of a membrane scaffold protein in nanodiscs was obtained by Rienstra and colleagues, through magic-angle spinning solid-state $\mathrm{NMR}^{32}$, and was quickly followed by acquisition of high-resolution 2D NMR signals for a large MP assembled in a nanodisc bilayer ${ }^{8,33}$. Many laboratories have recently reported impressive solution NMR of MPs in nano$\operatorname{discs}^{14,16,34,35}$. The pioneering work of Wagner and colleagues has provided the first complete atomic-resolution structures of an MP (OmpX) in a nanodisc bilayer ${ }^{14}$, which revealed significant differences between the structures of an MP in solution and in its native phospholipid environment.

Nanodiscs have also been used for individual distance measurements. For instance, EPR has been extensively used to monitor the
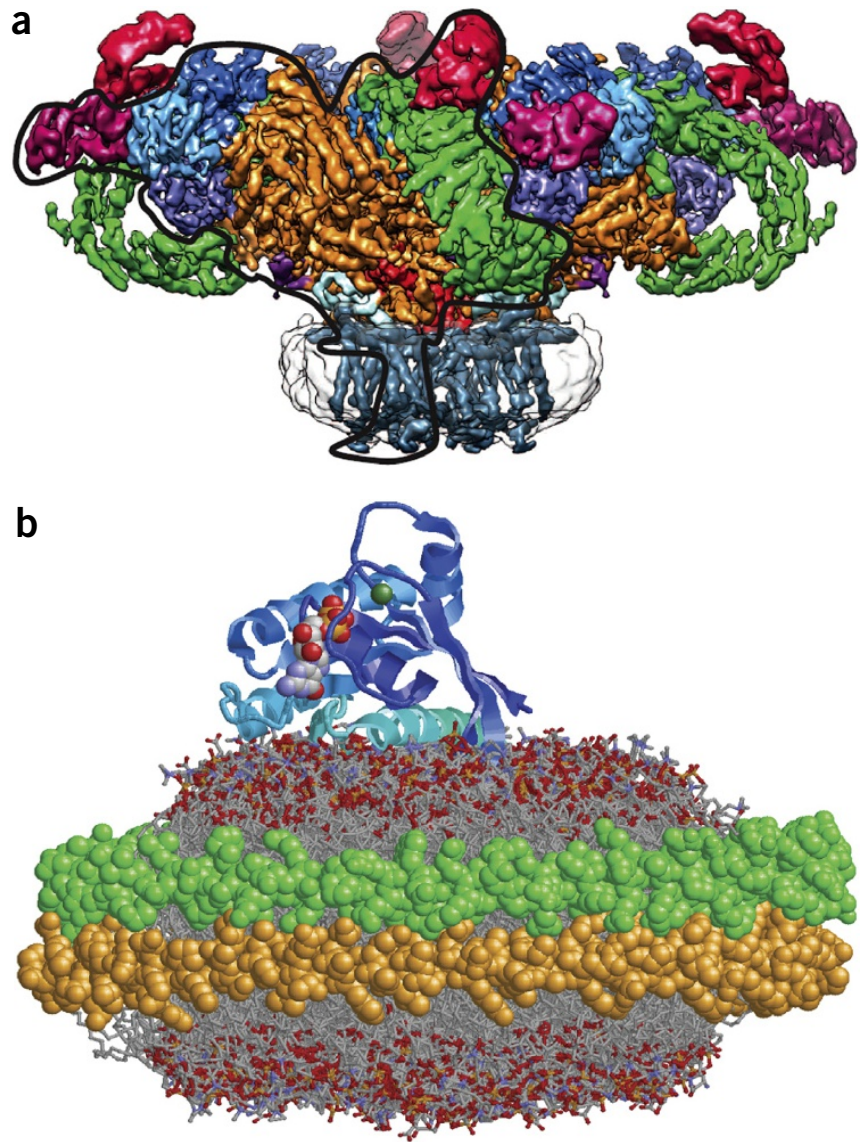

conformational changes and dynamics of ligand binding in the maltose transporter MalFGK 2 (ref. 36). EPR probes placed at selected sites on the target have allowed the observation of functionally important conformational changes in nanodiscs but not in detergentsolubilized protein. In another study, direct distance measurements using DEER spectroscopy have been used to monitor the conformational changes of a nanodisc-incorporated G-protein-coupled receptor (GPCR) upon binding of its signaling partner ${ }^{37}$.

The nanodisc as a controlled membrane surface. The membrane surface is the site for recruitment of many signaling proteins, and it is now viewed as an important player in dictating the structure of signaling molecules and the faithful formation of specific multiprotein complexes that are required for activity. Notably, nanodiscs have recently been used by investigators at the Frederick National Laboratory for Cancer Research to document the affinity of the oncogenic protein K-Ras4B for nanodisc surfaces ${ }^{9}$, and by Ikura and colleagues in solution NMR to define the conformational changes of K-Ras4B bound to nanodiscs ${ }^{38}$. Here, the NMR study of an MP in nanodiscs has provided important information about the orientation of the protein with respect to the lipid bilayer, specific proteinlipid membrane interactions involved in molecular recognition and the dynamics of small-molecule-binding sites at the protein-lipid interface. Other systems that have used nanodiscs to reveal specific lipid compositions that are necessary to recruit proteins to the membrane surface include studies of the blood coagulation cascade ${ }^{10}$ and the activation of integrins ${ }^{28}$. Nanodiscs have been extensively used to reveal the role of specific phospholipid types in MP function. Published work includes documenting the upshift of redox potential 
of redox transfer proteins due to the presence of negatively charged lipids ${ }^{39}$ and the critical role of cardiolipin in the assembly, activity and production of reactive oxygen species by the mitochondrial respiratory complex $\mathrm{II}^{40}$. The ability to precisely control the mixture of lipids in nanodiscs has enabled determination of lipid specificity in the binding of blood coagulation factors ${ }^{10}$, integrin activators ${ }^{28}$ and Ras oncogenes ${ }^{38}$.

$\mathrm{X}$-ray and neutron scattering. Both the structure of, and phase transition in, lipid-bilayer nanodiscs have been characterized with SAXS, fluorescence and calorimetry ${ }^{2,41}$. SAXS analysis of several MPs in nanodiscs has been used to confirm faithful incorporation in the lipid bilayer and to characterize the topology of the target protein. Examples include bR trimers ${ }^{4}$, curdlan synthase ${ }^{42}$ and cytochrome P450 (ref. 43). The latter reference has provided the first demonstration of the potential of $a b$ initio reconstruction of a low-resolution MP structure based solely on SAXS data. Such analysis can be significantly improved and refined if SAXS data are combined with SANS data collected with various contrasting methods, such as partially deuterated solvent and lipids ${ }^{44}$, made possible by contrast matching to render the scaffold protein and lipid invisible.

Neutron reflectometry has been used to directly measure large conformational changes of MPs in nanodiscs, for example, the redox-dependent movement needed for efficient electron transfer by NADPH cytochrome P450 reductase ${ }^{45}$. The different conformations of monomers and dimers of vertebrate visual rhodopsins reconstituted in nanodiscs have been directly monitored during photoactivation, through high-angle X-ray scattering ${ }^{46}$. Comparison with the X-ray scattering calculated for the known structures of photocycle intermediates has suggested that a dimeric form of rhodopsin is present in the membrane, and this dimerization modulates the observed functionally important structural changes ${ }^{46}$.

\section{Single-molecule measurements}

Single-molecule studies are being increasingly used to avoid masking details of protein function when only ensemble averages are measured. Nanodiscs are ideally suited for this type of investigation because they can be affixed to a surface without impairing subtle conformational changes in MPs. Either scaffold proteins or lipids have been labeled with affinity tags to immobilize nanodiscs on a surface while allowing access to both sides of the bilayer for the association of substrates or signaling partners. This label-free approach avoids suggestions that derivatization of the target alters its function or conformation. Single-molecule nanodisc studies have been reported for cytochrome P450 reductase ${ }^{47}$, cytochrome P450 CYP3A4 (ref. 48) and the $\beta 2$-adrenergic receptor ${ }^{49}$. The natural affinity of lipids for atomically flat silicon oxide or mica surfaces allows nanodiscs to sit flat, thus enabling atomic force microscopy to directly measure the depth of insertion of proteins in the bilayer ${ }^{50}$ and linear dichroism optical spectroscopy to quantify the orientation of a target protein relative to the nanodisc surface ${ }^{51}$

\section{Spectroscopic assay techniques}

In comparison to other membrane mimetics, nanodiscs provide substantial advantages for optical spectroscopy because of their low viscosity and lack of turbidity. These qualities allow the use of rapid mixing methods, such as stopped or continuous flow, for monitoring structural and functional changes on a millisecond time scale, while preserving the target MP in a lipid bilayer environment ${ }^{52}$. Laser flash techniques have been used to study the monomer and trimer of bacteriorhodopsin $(\mathrm{bR})^{4,53}$ and various rhodopsins ${ }^{54,55}$.
Optical spectroscopy and rapid mixing have also been used to probe functional intermediates in human cytochrome P450, including stopped flow ${ }^{52}$ and resonance Raman spectroscopy ${ }^{56}$. In all cases, biophysical studies have been enabled by the high stability and structural homogeneity of monomeric proteins and their functional complexes with redox partners in nanodiscs ${ }^{57,58}$.

Other ligand binding events, such as the kinetics of oxygen binding and autoxidation, have also shown clean monoexponential behavior when measured for monomers in nanodiscs, as opposed to solution aggregates. As with rhodopsin monomer and dimer preparations ${ }^{59,60}$, nanodiscs have helped to avoid ambiguities in the interpretation of experimental data and to assign the observed functional properties of the main drug-metabolizing human P450 enzyme CYP3A4 to the intrinsic behavior of its monomeric form. Allosteric regulation in this enzyme is determined by the properties of the peripheral highaffinity binding site at the protein-lipid interface ${ }^{61}$. Importantly, this binding pocket is formed when CYP3A4 is incorporated into nanodisc membranes and cannot be probed in detergentsolubilized preparations.

New enzyme intermediates can be trapped with nanodiscs. For instance, resonance Raman spectroscopy of human CYP17A1, made possible by cryogenic methods, has revealed a previously unobserved functional intermediate in the last step of androstenedione biosynthesis, thus answering a long-standing question as to the mechanism of this important drug target ${ }^{56}$.

\section{Other applications of nanodiscs}

Antibodies are extensively used in the study of MPs, often as an aid in crystallization or in the development of therapeutic agents. Raising antibodies against MPs is inherently difficult because an aggregated state can both occlude the antigenic site and lower the overall immune response. In vitro phage- and yeast-display technologies provide substantial advantages yet still require an MP in its native conformation, ideally in the absence of detergents. Pioneering work in the Kossiakoff laboratory has used nanodiscs to develop protocols for competitive and subtractive selection in phage-display approaches ${ }^{62}$.

Many MPs operate in higher-order complexes, ranging from homo- or heterodimers to large supramolecular complexes containing many subunits. Large molecular machines, such as cytochrome oxidases $^{63}$, the photosynthetic reaction center ${ }^{64}$ and peptide translocases ${ }^{25}$, can be functionally self-assembled into nanodiscs. In the case of signaling entities, individual monomers or oligomers of specific composition can be prepared, thus allowing the measurement of activity as a function of oligomerization state. For example, the first demonstrations that a monomeric GPCR has full signaling activity used nanodiscs containing either a rhodopsin monomer or dimer ${ }^{59,60}$. To study complex signaling pathways, for example those involved in chemotaxis signaling, nanodiscs have been used to separate the roles of dimers and trimers of dimers in specific activities ${ }^{65}$. Other examples of controlled oligomerization through the use of nanodiscs include studies of many rhodopsins ${ }^{55,66}$ and the plasma membrane $\mathrm{H}^{+}$-ATPase from Arabidopsis ${ }^{67}$. Because nanodisc assemblies have high stability, these fractions can be isolated and purified to homogeneity through size-exclusion chromatography or density gradient ultracentrifugation ${ }^{3}$.

\section{Conclusion and perspectives}

The use of nanodiscs is rapidly spreading, as facilitated by ready access to the genes encoding membrane scaffold proteins from AddGene and purified proteins from Sigma or BioNanoCon. In addition to the established applications of nanodisc technology, some recent 
developments have suggested new ideas, such as the generation of therapeutic antibodies against antigens incorporated into nanodiscs ${ }^{68}$ and various in vivo uses of nanodiscs, including drug delivery ${ }^{69}$, imaging ${ }^{70}$ and vaccine development. An exciting application is the ability to generate soluble MP libraries that faithfully represent a starting MP composition, with individual nanodiscs carrying a specific target ${ }^{5}$. By allowing high-throughput screening of MPs, nanodiscs can potentially facilitate the discovery of new approaches to therapeutic intervention.

\section{ACKNOWLEDGMENTS}

Nanodisc research in our laboratory is supported by the National Institutes of Health.

\section{COMPETING FINANCIAL INTERESTS}

The authors declare no competing financial interests.

Reprints and permissions information is available online at http://www.nature.com/ reprints/index.html.

1. Bayburt, T.H., Grinkova, Y.V. \& Sligar, S.G. Self-assembly of discoidal phospholipid bilayer nanoparticles with membrane scaffold proteins. Nano Lett. 2, 853-856 (2002).

2. Denisov, I.G., Grinkova, Y.V., Lazarides, A.A. \& Sligar, S.G. Directed self-assembly of monodisperse phospholipid bilayer Nanodiscs with controlled size. J. Am. Chem. Soc. 126, 3477-3487 (2004).

3. Bayburt, T.H. \& Sligar, S.G. Membrane protein assembly into Nanodiscs. FEBS Lett. 584, 1721-1727 (2010).

4. Bayburt, T.H., Grinkova, Y.V. \& Sligar, S.G. Assembly of single bacteriorhodopsin trimers in bilayer nanodiscs. Arch. Biochem. Biophys. 450, 215-222 (2006).

5. Wilcox, K.C. et al. Nanoscale synaptic membrane mimetic allows unbiased high throughput screen that targets binding sites for Alzheimer's-associated $A \beta$ oligomers. PLOS One 10, e0125263 (2015)

6. Dörr, J.M. et al. The styrene-maleic acid copolymer: a versatile tool in membrane research. Eur. Biophys. J. 45, 3-21 (2016).

7. Planchard, N. et al. The use of amphipols for solution NMR studies of membrane proteins: advantages and constraints as compared to other solubilizing media. J. Membr. Biol. 247, 827-842 (2014).

8. Kijac, A.Z., Li, Y., Sligar, S.G. \& Rienstra, C.M. Magic-angle spinning solid-state NMR spectroscopy of nanodisc-embedded human CYP3A4. Biochemistry 46, 13696-13703 (2007).

9. Gillette, W.K. et al. Farnesylated and methylated KRAS4b: high yield production of protein suitable for biophysical studies of prenylated protein-lipid interactions. Sci. Rep. 5, 15916 (2015)

10. Shaw, A.W., Pureza, V.S., Sligar, S.G. \& Morrissey, J.H. The local phospholipid environment modulates the activation of blood clotting. J. Biol. Chem. 282, 6556-6563 (2007).

11. Ritchie, T.K., Kwon, H. \& Atkins, W.M. Conformational analysis of human ATP-binding cassette transporter ABCB1 in lipid nanodiscs and inhibition by the antibodies MRK16 and UIC2. J. Biol. Chem. 286, 39489-39496 (2011).

12. Proverbio, D., Henrich, E., Orbán, E., Dötsch, V. \& Bernhard, F. Membrane protein quality control in cell-free expression systems: tools, strategies and case studies. in Membrane Proteins Production for Structural Analysis (ed. Mus-Veteau, I.) 45-70 (Springer, 2014)

13. Catoire, L.J., Warnet, X.L. \& Warschawski, D.E. Micelles, bicelles, amphipols, nanodiscs, liposomes, or intact cells: the hitchhiker's guide to the study of membrane proteins by NMR. in Membrane Proteins Production for Structural Analysis (ed. Mus-Veteau, I.) 315-345 (Springer, 2014).

14. Hagn, F., Etzkorn, M., Raschle, T. \& Wagner, G. Optimized phospholipid bilayer nanodiscs facilitate high-resolution structure determination of membrane proteins. J. Am. Chem. Soc. 135, 1919-1925 (2013).

15. Malhotra, K. \& Alder, N.N. Advances in the use of nanoscale bilayers to study membrane protein structure and function. Biotechnol. Genet. Eng. Rev. 30, 79-93 (2014).

16. Morgado, L., Zeth, K., Burmann, B.M., Maier, T. \& Hiller, S. Characterization of the insertase BamA in three different membrane mimetics by solution NMR spectroscopy. J. Biomol. NMR 61, 333-345 (2015)

17. Shenkarev, Z.O. et al. Lipid-protein nanodiscs offer new perspectives for structural and functional studies of water-soluble membrane-active peptides. Acta Naturae $\mathbf{6}$, 84-94 (2014)

18. Wang, X., Mu, Z., Li, Y., Bi, Y. \& Wang, Y. Smaller nanodiscs are suitable for studying protein lipid interactions by solution NMR. Protein J. 34, 205-211 (2015).

19. Zhou, H.X. \& Cross, T.A. Influences of membrane mimetic environments on membrane protein structures. Annu. Rev. Biophys. 42, 361-392 (2013).

20. Akkaladevi, N. et al. Following natures lead: on the construction of membraneinserted toxins in lipid bilayer nanodiscs. J. Membr. Biol. 248, 595-607 (2015).

21. Efremov, R.G., Leitner, A., Aebersold, R. \& Raunser, S. Architecture and conformational switch mechanism of the ryanodine receptor. Nature 517, 39-43 (2015).
22. Chung, K.Y., Day, P.W., Vélez-Ruiz, G., Sunahara, R.K. \& Kobilka, B.K. Identification of GPCR-interacting cytosolic proteins using HDL particles and mass spectrometrybased proteomic approach. PLoS One 8, e54942 (2013).

23. Rasmussen, S.G. et al. Structure of a nanobody-stabilized active state of the $\beta_{2}$ adrenoceptor. Nature 469, 175-180 (2011).

24. Dominik, P.K. et al. Conformational chaperones for structural studies of membrane proteins using antibody phage display with nanodiscs. Structure 24, 300-309 (2016).

25. Frauenfeld, J. et al. Cryo-EM structure of the ribosome-SecYE complex in the membrane environment. Nat. Struct. Mol. Biol. 18, 614-621 (2011).

26. Gogol, E.P. et al. Three dimensional structure of the anthrax toxin translocon-lethal factor complex by cryo-electron microscopy. Protein Sci. 22, 586-594 (2013).

27. Katayama, H. et al. Three-dimensional structure of the anthrax toxin pore inserted into lipid nanodiscs and lipid vesicles. Proc. Natl. Acad. Sci. USA 107, 3453-3457 (2010).

28. Ye, F. et al. Recreation of the terminal events in physiological integrin activation J. Cell Biol. 188, 157-173 (2010).

29. Grushin, K., Miller, J., Dalm, D. \& Stoilova-McPhie, S. Factor VIII organisation on nanodiscs with different lipid composition. Thromb. Haemost. 113, 741-749 (2015).

30. Zhang, P. et al. An isoform-specific myristylation switch targets type II PKA holoenzymes to membranes. Structure 23, 1563-1572 (2015).

31. Matthies, D. et al. Cryo-EM structures of the magnesium channel CorA reveal symmetry break upon gating. Cell 164, 747-756 (2016).

32. Li, Y., Kijac, A.Z., Sligar, S.G. \& Rienstra, C.M. Structural analysis of nanoscale self-assembled discoidal lipid bilayers by solid-state NMR spectroscopy. Biophys. J. 91, 3819-3828 (2006).

33. Ding, Y., Fujimoto, L.M., Yao, Y. \& Marassi, F.M. Solid-state NMR of the Yersinia pestis outer membrane protein Ail in lipid bilayer nanodiscs sedimented by ultracentrifugation. J. Biomol. NMR 61, 275-286 (2015).

34. Kucharska, I., Edrington, T.C., Liang, B. \& Tamm, L.K. Optimizing nanodiscs and bicelles for solution NMR studies of two $\beta$-barrel membrane proteins. J. Biomol. NMR 61, 261-274 (2015).

35. Mineev, K.S., Goncharuk, S.A., Kuzmichev, P.K., Vilar, M. \& Arseniev, A.S. NMR dynamics of transmembrane and intracellular domains of p75NTR in lipid-protein nanodiscs. Biophys. J. 109, 772-782 (2015).

36. Alvarez, F.J. et al. Full engagement of liganded maltose-binding protein stabilizes a semi-open ATP-binding cassette dimer in the maltose transporter. Mol. Microbiol. 98, 878-894 (2015)

37. Kang, Y. et al. Crystal structure of rhodopsin bound to arrestin by femtosecond X-ray laser. Nature 523, 561-567 (2015).

38. Mazhab-Jafari, M.T. et al. Oncogenic and RASopathy-associated K-RAS mutations relieve membrane-dependent occlusion of the effector-binding site. Proc. Natl. Acad. Sci. USA 112, 6625-6630 (2015).

39. Das, A. \& Sligar, S.G. Modulation of the cytochrome P450 reductase redox potential by the phospholipid bilayer. Biochemistry 48, 12104-12112 (2009).

40. Schwall, C.T., Greenwood, V.L. \& Alder, N.N. The stability and activity of respiratory Complex II is cardiolipin-dependent. Biochim. Biophys. Acta. 1817, 1588-1596 (2012).

41. Denisov, I.G., McLean, M.A., Shaw, A.W., Grinkova, Y.V. \& Sligar, S.G. Thermotropic phase transition in soluble nanoscale lipid bilayers. J. Phys. Chem. B 109, 15580-15588 (2005)

42. Periasamy, A. et al. Cell-free protein synthesis of membrane (1,3)- $\beta$-D-glucan (curdlan) synthase: Co-translational insertion in liposomes and reconstitution in nanodiscs. Biochim. Biophys. Acta. 1828, 743-757 (2013).

43. Skar-Gislinge, N. et al. Small-angle scattering determination of the shape and localization of human cytochrome P450 embedded in a phospholipid nanodisc environment. Acta Crystallogr. D Biol. Crystallogr. 71, 2412-2421 (2015).

44. Maric, S. et al. Stealth carriers for low-resolution structure determination of membrane proteins in solution. Acta Crystallogr. D Biol. Crystallogr. 70, 317-328 (2014).

45. Wadsäter, M. et al. Monitoring shifts in the conformation equilibrium of the membrane protein cytochrome P450 reductase (POR) in nanodiscs. J. Biol. Chem. 287, 34596-34603 (2012).

46. Imamoto, Y., Kojima, K., Oka, T., Maeda, R. \& Shichida, Y. Helical rearrangement of photoactivated rhodopsin in monomeric and dimeric forms probed by high-angle X-ray scattering. Photochem. Photobiol. Sci. 14, 1965-1973 (2015).

47. Laursen, T. et al. Single molecule activity measurements of cytochrome P450 oxidoreductase reveal the existence of two discrete functional states. ACS Chem. Biol. 9, 630-634 (2014).

48. Nath, A., Koo, P.K., Rhoades, E. \& Atkins, W.M. Allosteric effects on substrate dissociation from cytochrome P450 3A4 in nanodiscs observed by ensemble and single-molecule fluorescence spectroscopy. J. Am. Chem. Soc. 130, 15746-15747 (2008).

49. Lamichhane, R. et al. Single-molecule view of basal activity and activation mechanisms of the G protein-coupled receptor $\beta 2$ AR. Proc. Natl. Acad. Sci. USA 112, 14254-14259 (2015).

50. Bayburt, T.H. \& Sligar, S.G. Single-molecule height measurements on microsomal cytochrome P450 in nanometer-scale phospholipid bilayer disks. Proc. Natl. Acad. Sci. USA 99, 6725-6730 (2002).

51. Baylon, J.L., Lenov, I.L., Sligar, S.G. \& Tajkhorshid, E. Characterizing the membranebound state of cytochrome P450 3A4: structure, depth of insertion, and orientation. J. Am. Chem. Soc. 135, 8542-8551 (2013). 
52. Denisov, I.G., Grinkova, Y.V., McLean, M.A. \& Sligar, S.G. The one-electron autoxidation of human cytochrome P450 3A4. J. Biol. Chem. 282, 26865-26873 (2007).

53. Johnson, P.J.M. et al. The photocycle and ultrafast vibrational dynamics of bacteriorhodopsin in lipid nanodiscs. Phys. Chem. Chem. Phys. 16, 21310 21320 (2014)

54. Tsukamoto, H., Szundi, I., Lewis, J.W., Farrens, D.L. \& Kliger, D.S. Rhodopsin in nanodiscs has native membrane-like photointermediates. Biochemistry 50, 5086-5091 (2011).

55. Ranaghan, M.J., Schwall, C.T., Alder, N.N. \& Birge, R.R. Green proteorhodopsin reconstituted into nanoscale phospholipid bilayers (nanodiscs) as photoactive monomers. J. Am. Chem. Soc. 133, 18318-18327 (2011).

56. Mak, P.J., Gregory, M.C., Denisov, I.G., Sligar, S.G. \& Kincaid, J.R. Unveiling the crucial intermediates in androgen production. Proc. Natl. Acad. Sci. USA 112, 15856-15861 (2015)

57. Denisov, I.G., Baas, B.J., Grinkova, Y.V. \& Sligar, S.G. Cooperativity in cytochrome P450 3A4: linkages in substrate binding, spin state, uncoupling, and product formation. J. Biol. Chem. 282, 7066-7076 (2007).

58. Khatri, Y., Gregory, M.C., Grinkova, Y.V., Denisov, I.G. \& Sligar, S.G. Active site proton delivery and the lyase activity of human CYP17A1. Biochem. Biophys. Res. Commun. 443, 179-184 (2014).

59. Bayburt, T.H., Leitz, A.J., Xie, G., Oprian, D.D. \& Sligar, S.G. Transducin activation by nanoscale lipid bilayers containing one and two rhodopsins. J. Biol. Chem. 282, 14875-14881 (2007).

60. Bayburt, T.H. et al. Monomeric rhodopsin is sufficient for normal rhodopsin kinase (GRK1) phosphorylation and arrestin-1 binding. J. Biol. Chem. 286, 1420-1428 (2011).
61. Denisov, I.G., Grinkova, Y.V., Baylon, J.L., Tajkhorshid, E. \& Sligar, S.G. Mechanism of drug-drug interactions mediated by human cytochrome P450 CYP3A4 monomer. Biochemistry 54, 2227-2239 (2015).

62. Dominik, P.K. \& Kossiakoff, A.A. Phage display selections for affinity reagents to membrane proteins in nanodiscs. Methods Enzymol. 557, 219-245 (2015)

63. Näsvik Öjemyr, L., von Ballmoos, C., Gennis, R.B., Sligar, S.G. \& Brzezinski, P. Reconstitution of respiratory oxidases in membrane nanodiscs for investigation of proton-coupled electron transfer. FEBS Lett. 586, 640-645 (2012).

64. Ham, M.-H. et al. Photoelectrochemical complexes for solar energy conversion that chemically and autonomously regenerate. Nat. Chem. 2, 929-936 (2010).

65. Li, M. \& Hazelbauer, G.L. Selective allosteric coupling in core chemotaxis signaling complexes. Proc. Natl. Acad. Sci. USA 111, 15940-15945 (2014).

66. Moers, K. et al. Modified lipid and protein dynamics in nanodiscs. Biochim. Biophys. Acta. 1828, 1222-1229 (2013)

67. Justesen, B.H. et al. Active plasma membrane P-type $\mathrm{H}^{+}$-ATPase reconstituted into nanodiscs is a monomer. J. Biol. Chem. 288, 26419-26429 (2013).

68. Reichart, T.M. et al. Trimerization of the HIV transmembrane domain in lipid bilayers modulates broadly neutralizing antibody binding. Angew. Chem. Int. Ed. Engl. 55 2688-2692 (2016).

69. Numata, M. et al. Nanodiscs as a therapeutic delivery agent: inhibition of respiratory syncytial virus infection in the lung. Int. J. Nanomedicine 8 1417-1427 (2013).

70. Carney, C.E. et al. Nanodiscs as a modular platform for multimodal MR-optical imaging. Bioconjug. Chem. 26, 899-905 (2015). 